2019-07

\title{
Coast/breakwater-integrated OWC: A theoretical model
}

\section{Zheng, Siming}

http://hdl.handle.net/10026.1/13733

10.1016/j.marstruc.2019.04.001

Marine Structures

Elsevier

All content in PEARL is protected by copyright law. Author manuscripts are made available in accordance with publisher policies. Please cite only the published version using the details provided on the item record or document. In the absence of an open licence (e.g. Creative Commons), permissions for further reuse of content should be sought from the publisher or author. 
1 Title: Coast/breakwater-integrated OWC: a theoretical model

2

3

4
Siming Zheng ${ }^{\mathrm{a}}$, Yongliang Zhang ${ }^{\mathrm{b}}$, Gregorio Iglesias ${ }^{\mathrm{a}, \mathrm{c}}$

\section{Author names and affiliations:}

Siming Zheng

E-mail address: siming.zheng@plymouth.ac.uk

Yongliang Zhang

E-mail address: yongliangzhang@tsinghua.edu.cn

Gregorio Iglesias

E-mail address: gregorio.iglesias@ucc.ie

a School of Engineering, University of Plymouth, Drake Circus, Plymouth PL4 8AA, United Kingdom

b State Key Laboratory of Hydroscience and Engineering, Tsinghua University, Beijing, 100084, China

c Centre for Marine and Renewable Energy Ireland (MaREI), Environmental Research Institute \& School of Engineering, University College Cork, Ireland

https://doi.org/10.1016/j.marstruc.2019.04.001

Received 19 October 2018; Received in revised form 3 January 2019; Accepted 6 April 2019

Available online 15 April 2019 


\begin{abstract}
Integrating wave energy converters into coastal structures such as breakwaters, seawalls or jetties not only offers benefits in terms of construction costs but also improves wave energy extraction. In this paper a novel theoretical model based on linear potential flow theory is developed to study the performance of an oscillating water column (OWC) integrated into a vertical structure in water of finite water depth. The model has three fundamental advantages relative to previous works: no thin-wall restriction (the thickness of the OWC chamber wall is considered), no singularities, and far fewer truncating terms in the eigen-function expansions. The OWC chamber is a vertical cylinder semi-embedded in the structure with a submerged, open bottom. As water waves impinge on the structure, the water column in the chamber oscillates and drives an air turbine installed at the chamber top to extract wave power. Using linear wave theory, the velocity potential in the water domain is decomposed into scattering and radiation potentials whose unknown coefficients are determined by the eigen-function matching method. Upon successful validation, the model is used to investigate the influence of the thickness of the chamber wall and the radius and submergence of the chamber on wave power absorption.
\end{abstract}

Keywords: Oscillating Water Column; Breakwater-integrated OWC; Wave energy; Potential flow; Excitation volume flow; Hydrodynamic coefficients

\title{
1. Introduction
}

A large number of wave energy conversion concepts have been proposed since 1970s, which can be roughly classified as: oscillating water column (OWC) (e.g., [1, 2]), point absorber (e.g., [3]), attenuator (e.g., [4]), oscillating wave surge converter (e.g., [5]), overtopping (e.g., [6]), and others. Despite the large number of concepts proposed and investigated so far, only a few wave energy converters (WECs) have been tested at a large scale, and even fewer have achieved the fully commercial stage [7,8]. The challenges in bringing WECs to the market include: high cost of construction, installation and maintenance; negative environmental impact; poor reliability; and low power extraction efficiency $[9,10]$. It is not easy to solve all these problems concurrently since some of them might be in conflict - and therein lie the challenges. For example, the improvement of the reliability of WECs generally results in an increased cost of construction; to enhance the power capture efficiency more sophisticated systems are typically necessary, but this very sophistication is generally detrimental to the overall cost and survivability of the system. The integration of a WEC into a marine structure, e.g., a breakwater, as opposed to its stand-alone deployment in the open sea is an effective means to overcome a number of these challenges and significantly increase the attractiveness of wave power exploitation [9, 11]. The integration not only offers benefits in terms of shared costs of construction, but also improves the robustness of the WEC and minimizes its environmental impact. Additionally, thanks to the wave power absorbed by the WEC, wave reflection at the structure is diminished, which is often advantageous from the points of view of coastal protection and non-interference with shipping. The synergies between wave energy and marine structures have been investigated in a number of works, e.g., integration of an array of WECs with a breakwater [12], integration of an OWC into an offshore wind turbine [13] or breakwater [14].

Among wave energy conversion technologies, OWC systems are especially simple, for the only 
moving mechanical part is an air turbine/generator located above the water; therefore, OWC-breakwater integration has received considerable attention [9]. Evans and Porter [15] developed a theoretical model to simulate a two-dimensional (2D) OWC device composed of a thin vertical surface-piercing barrier in front of a vertical wall. An integral equation for the horizontal velocity across the gap under the thin barrier was adopted to deal with the singular behaviour in the velocity field. Theoretical results showed that increasing the distance between the barrier and the wall decreased the frequency at which resonance occurred. Later, Morris-Thomas, Irvin [16] examined effect of the front barrier geometry on the performance of the OWC experimentally. The hydrodynamic efficiency in short waves was found to decrease with the increase of the barrier's submergence or thickness. More recently, the impact of the underwater lips of an offshore OWC device in terms of both thickness and submergence was investigated by Elhanafi, Fleming [17] with a two-dimensional computational fluid dynamics (CFD) model. By selecting the optimal combination of the submergence and thickness of the lips, a peak efficiency exceeding 0.79 was achieved, much larger than the 0.3 for a device with simpler, typical geometry. Other aspects such as the role of the turbine Power Take-Off (PTO) system and the environmental conditions in the power extraction of an onshore or bottom-fixed, breakwater-integrated OWC have also been investigated. Sheng, Alcorn [18] proposed a numerical method based on potential flow theory to assess the primary energy conversion of two generic OWC WECs (one bottom fixed and another floating). The hydrodynamics and thermodynamics with consideration of the air compressibility for different types of the air turbine PTOs (i.e., Wells turbine, impulse turbines and bi-radial turbines) were coupled in the time-domain, and the numerical results appeared accurate enough for the OWC power extraction assessment, especially for the bottom-fixed OWC. López and Iglesias [19] developed a virtual laboratory based on artificial neural networks that can be employed to obtain the pneumatic efficiency of a given OWC under specific wave condition, tidal level and turbine damping. Physical model tests of these parameters were carried out as well [20]. In order to achieve an optimal energy transfer, Pereiras, López [21] described a methodology for matching a nonlinear turbine to the OWC chamber. Elhanafi, Fleming [22] adopted a CFD model to learn the impacts of both the PTO damping and incoming wave height on the performance of an onshore OWC. The reflection coefficient and the energy absorption coefficient generally increase and decrease with wave height. Research has also been directed towards other types of onshore or bottom-fixed, breakwater-integrated OWCs, e.g., the U-type OWC [23, 24] and the multi-chamber OWC [25]. Additionally, the integration of OWCs with floating breakwaters was considered by He, Huang [26], He, Leng [27].

The above studies are focused on 2D problems of OWC-breakwater integration. In contrast, there are few studies on its 3D aspects. For experimental work these require a wave basin rather than a flume, with a scale model of the breakwater as well as the OWC itself [28]. For numerical work, if the boundary element method is employed, the surfaces of the OWC device and the surrounding breakwater or coastline must be divided into elements [29]; if the finite element method is adopted, a numerical wave basin shall be established with the entire water volume discretized [30]. Due to the considerable experimental and computational cost, 3D studies of OWC-structure integration are not common. If the shapes of the structure and OWC are regular, theoretical models may be used to solve the 3D hydrodynamic problem. Martins-rivas and Mei [31] proposed a theoretical model based on the 3D wave radiation/diffraction theory and the usual method of eigen-function expansions to study wave power extraction from an OWC at the tip of a 
long and thin breakwater. The thin-walled OWC was represented by a hollow cylinder in their model, in which the method for solving the integral equation of Evans and Porter [15] was used to treat the singular behaviours in the velocity field beneath the thin wall of the OWC chamber. The linearized air compressibility in the chamber was taken into account as part of the PTO system. The effects of the radius and submergence of the OWC chamber, the air compressibility and the incident wave direction were investigated. It was found that the free surface outside was strongly dependent on the incident wave direction, whereas the power extracted was roughly insensitive to the incident direction. Subsequently, Martins-rivas and Mei [32] applied the same theoretical approach to a thin-walled OWC installed on a straight cliff-like coast. The performance of the OWC was found to strongly depend on the incident wave direction. Wave reflection at the coast could lead to up to a doubling in the power absorbed by the OWC. The role of either the radius or submergence of the OWC was not considered. This theoretical model was later applied by Lovas, Mei [33] to a vertical OWC at the tip of a general wedge-shaped coast.

In this context we propose a novel theoretical model based on linear potential flow theory to evaluate the hydrodynamic performance and power extraction of the OWC. This novel approach has three fundamental advantages relative to previous works. First, the thin-wall restriction is removed, i.e., the thickness of the OWC chamber wall is taken into consideration. Second, there is no singularity. Finally, far fewer truncating terms of the eigen-function expansions are required to obtain accurate results. The effects of wall thickness, radius and submergence of the OWC on power extraction can thus be investigated with the present model - as shown below.

The rest of the paper is organized as follows. Section 2 presents the relation between the PTO system and the hydrodynamic problem. The basic governing equation, the boundary conditions for wave scattering and radiation problems, and the expressions of the scattering and radiated velocity potentials in different regions of the water domain are developed in Section 3, alongside the method for solving the unknown coefficients. The expressions of excitation volume flow and hydrodynamic coefficients are derived in Section 4. The model validation can be found in Section 5. The influence of the radius, wall thickness and submergence of the OWC chamber on wave power absorption are investigated in Section 6. Finally, conclusions are drawn in Section 7.

\section{Mathematical model}

Consider an oscillating water column (OWC) installed on a vertical wall in a water domain of uniform depth $h$ (see Fig.1). The OWC chamber is composed of a vertical circular cylinder with a ring shape cross section and it is half embedded in the wall. The outer and inner radii of the OWC chamber are denoted as $R$ and $R_{\mathrm{i}}$, respectively. On the seaside, the chamber is open from a finite submergence, denoted as $d$, to the seabed. As water waves propagate in the direction of $\beta$, the water column enclosed by the OWC chamber oscillates and drives a Wells turbine (not plotted in Fig. 1) installed at the chamber top to extract wave power. 
151

152

153

154

155

156

157

158

159

160

161

162

163

164

165

166

167

168

169

170

171

172

173

174

175

176

177

178

179

180

181

182 (a)

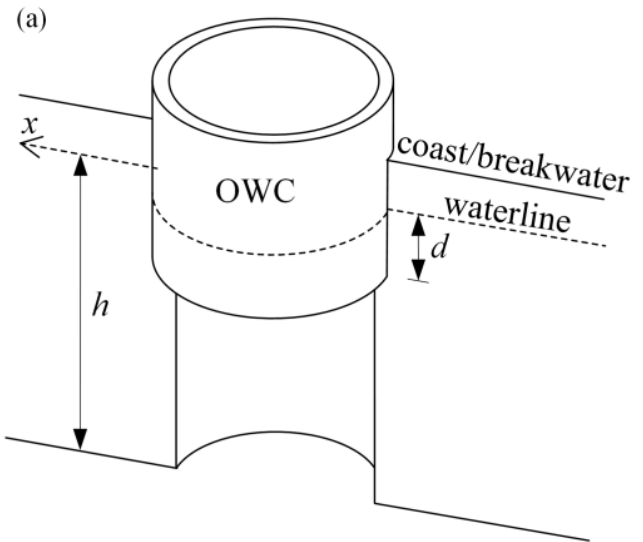

(b)

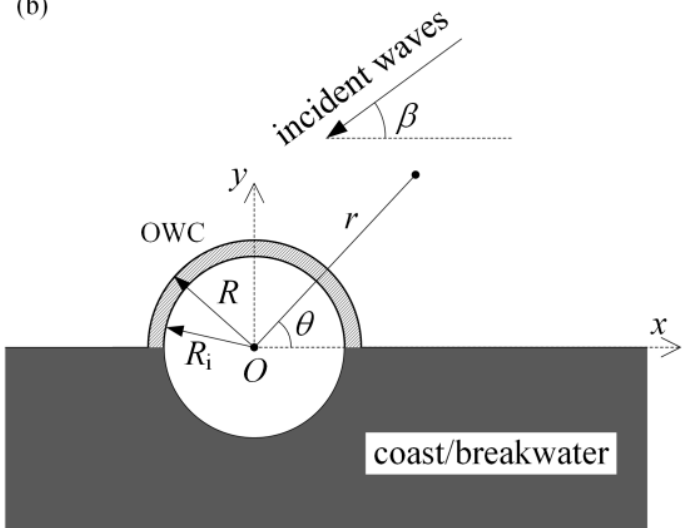

Fig. 1. Oscillating water column integrated into a coast/breakwater: (a) bird view; (b) top view.

As shown in Fig. 1, a general Cartesian coordinate system $O x y z$ is adopted with the $O z$ axis at the location of the symmetrical vertical axis of the OWC pointing upward and the $O x$ axis along the waterline at the coast/breakwater. A polar coordinate $(\mathrm{O} r \theta z)$ is defined as given in Fig. 1b.

Subjected to regular waves of angular frequency $\omega$ with small amplitude, the flow problems may be treated in the linear potential theory regime in the frequency domain based on the assumption that the fluid is isotropic, incompressible inviscid, and the time-harmonic flow is irrotational. The fluid motion can be described by the velocity potential $\operatorname{Re}\left[\Phi(r, \theta, z) \mathrm{e}^{-\mathrm{i} \omega t}\right]$, where $\Phi$ is a complex spatial velocity potential independent of time, $t$, and satisfies the Laplace equation, $\mathrm{i}$ represents imaginary unit. In a similar way, the air pressure inside the OWC chamber can be written as $\operatorname{Re}\left(p \mathrm{e}^{-\mathrm{i} \omega t}\right)$, where $p$ is the complex air pressure amplitude inside the OWC chamber.

The spatial velocity potential $\Phi$ can be decomposed into the wave spatial potential, $\Phi_{\mathrm{I}}$, which represents the wave field when the vertical wall without OWC (i.e., a flat wall) is subjected to monochromatic incident waves, the diffracted wave spatial potential $\Phi_{\mathrm{D}}$ induced by the existence of the OWC, and the radiated wave spatial potential as follows

$$
\Phi=\Phi_{\mathrm{I}}+\Phi_{\mathrm{D}}+p \Phi_{\mathrm{R}}
$$

where $\Phi_{\mathrm{R}}$ is the spatial velocity potential due to unit air pressure oscillation inside the OWC chamber. $\Phi_{\mathrm{I}}, \Phi_{\mathrm{D}}$, and $\Phi_{\mathrm{R}}$ all satisfy the Laplace equation, the boundary condition at the side wall of the coast/breakwater and the seabed boundary condition. Moreover, $\Phi_{\mathrm{D}}$ and $\Phi_{\mathrm{R}}$ must satisfy a radiation condition at infinite distance. Hereinafter, the sum of the incident and diffracted velocity potentials, which is the so-called scattering velocity potential (i.e., $\Phi_{\mathrm{S}}=\Phi_{\mathrm{I}}+\Phi_{\mathrm{D}}$ ), is adopted for the sake of simplicity.

Considering the air turbine employed in the OWC is an idealized lossless linear Wells turbine and assuming the mass flux through the Wells turbines is proportional to the chamber air pressure, following Sarmento and Falcão [34] and Martins-rivas and Mei [32], the complex air pressure amplitude, $p$, is related to the scattering and radiated velocity potentials by:

$$
\left[-\mathrm{i}\left(a_{\mathrm{PTO}}+a\right)+\left(c_{\mathrm{PTO}}+c\right)\right] p=Q_{\mathrm{e}},
$$

where $a_{\mathrm{PTO}}$ is used to take into account the effect of air compressibility, and can be expressed as $a$ Pто $=\omega V_{0} /\left(v^{2} \rho_{0}\right)$, in which $V_{0}$ is the air chamber volume, $v$ denotes the sound velocity in air and $\rho_{0}$ represents the static air density; $c_{\text {РTO }}$ is the damping of the PTO system depending on the 
rotational speed of turbine blades, the scales of turbine rotor, the design of turbines and $\rho_{0}$ as well; $Q_{\mathrm{e}}$, the so-called excitation volume flow, is the rate of upward displacement of the water surface inside the column contributed by the scattering potential:

$$
Q_{\mathrm{e}}=\left.\int_{0}^{2 \pi} \int_{0}^{R_{\mathrm{i}}} \frac{\partial \Phi_{\mathrm{S}}}{\partial z}\right|_{z=0} r \mathrm{~d} r \mathrm{~d} \theta
$$

$c$ and $a$ are hydrodynamic coefficients that can be derived from the volume flow inside the column induced by the radiated potential (i.e., $Q_{\mathrm{R}}$ ),

$$
-(c-\mathrm{i} a)=Q_{\mathrm{R}}=\left.\int_{0}^{2 \pi} \int_{0}^{R_{\mathrm{i}}} \frac{\partial \Phi_{\mathrm{R}}}{\partial z}\right|_{z=0} r \mathrm{~d} r \mathrm{~d} \theta .
$$

The time-averaged power extraction by the PTO system (i.e., the Wells turbine), $P$, can be calculated by:

$$
P=\frac{c_{\text {PTO }}}{2}|p|^{2}=\frac{c_{\text {PTO }}}{2} \frac{\left|Q_{\mathrm{e}}\right|^{2}}{\left(c+c_{\text {PTO }}\right)^{2}+\left(a+a_{\text {PTO }}\right)^{2}} .
$$

The efficiency of wave power extraction is generally expressed by the relative wave capture width

$$
\eta=\frac{k P}{P_{\text {in }}}=\frac{2 k P}{\rho g A^{2} c_{\mathrm{g}}}
$$

where $P_{\text {in }}$ is the incident wave energy per unit width of the wave front; $c_{g}$ is the group velocity of the incident wave.

Note that $Q_{\mathrm{e}}, c$ and $a$ are fundamental for evaluating the performance of the OWC. In order to obtain these parameters, wave scattering and radiation problems, i.e., $\Phi_{\mathrm{S}}$ and $\Phi_{\mathrm{R}}$, should be solved first.

\section{Solution to scattering/radiated potentials}

The governing equation and the boundary conditions for $\Phi_{\chi}(\chi=\mathrm{S}, \mathrm{R})$ can be written as follows:

$$
\frac{\partial \Phi_{\chi}}{\partial z}=0, \quad z=-h
$$

$$
\frac{\partial \Phi_{\chi}}{\partial z}=0, \quad R_{\mathrm{i}} \leq r \leq R, \quad z=-d, 0<\theta<\pi,
$$

$$
\frac{\partial \Phi_{\chi}}{\partial z}-\frac{\omega^{2}}{g} \Phi_{\chi}=0, \quad r \geq R, \quad z=0,0<\theta<\pi
$$

$$
\frac{\partial \Phi_{\chi}}{\partial z}-\frac{\omega^{2}}{g} \Phi_{\chi}=\left\{\begin{array}{ll}
0, & \chi=\mathrm{S} \\
\frac{\mathrm{i} \omega}{\rho g}, & \chi=\mathrm{R}
\end{array}, \quad r \leq R_{\mathrm{i}}, \quad z=0, \quad 0 \leq \theta \leq 2 \pi,\right.
$$




$$
\frac{\partial \Phi_{\chi}}{\partial \theta}=0, \quad r>R,-h \leq z \leq 0, \theta=0, \pi
$$

$$
\frac{\partial \Phi_{\chi}}{\partial \theta}=0, \quad R_{\mathrm{i}}<r<R, \quad-h \leq z \leq-d, \quad \theta=0, \pi,
$$

$$
\frac{\partial \Phi_{\chi}}{\partial r}=0, \quad r=R,-d \leq z \leq 0,0 \leq \theta \leq \pi
$$

$$
\frac{\partial \Phi_{\chi}}{\partial r}=0, \quad r=R_{\mathrm{i}}, \quad-d \leq z \leq 0,0 \leq \theta \leq 2 \pi
$$

$$
\frac{\partial \Phi_{\chi}}{\partial r}=0, \quad r=R_{\mathrm{i}}, \quad-h \leq z \leq-d, \pi \leq \theta \leq 2 \pi,
$$

212

where $\rho$ is the water density and $g$ represents the gravity acceleration.

The entire fluid domain can be divided into three regions: I, inner region enclosed by the OWC, i.e., $r \leq R_{\mathrm{i}}, 0 \leq \theta \leq 2 \pi,-h \leq z \leq 0$; II, ring region beneath the OWC chamber, i.e., $R_{\mathrm{i}} \leq r \leq R, 0 \leq \theta \leq \pi,-h \leq z \leq-d$; III, outside region, i.e., $r \geq R, 0 \leq \theta \leq \pi,-h \leq z \leq 0$. $\Phi_{\chi}(\chi=\mathrm{S}, \mathrm{R})$ in these three regions are denoted as $\Phi_{\chi}^{\text {in }}, \Phi_{\chi}^{\text {ring }}$ and $\Phi_{\chi}^{\text {out }}$, respectively.

\subsection{Expressions of scattering/radiated potentials in different regions}

In different regions, with the application of the method of separation of variables, $\Phi_{\chi}(\chi=\mathrm{S}, \mathrm{R})$ can be expressed by orthogonal series as follows [35, 36]:

$\mathrm{I}$, inner region

$$
\Phi_{\chi}^{\mathrm{in}}(r, \theta, z)=\sum_{m=-\infty}^{\infty} \sum_{l=0}^{\infty} \frac{\tilde{I}_{m}\left(k_{l} r\right)}{k_{l} \tilde{I}_{m}^{\prime}\left(k_{l} R_{\mathrm{i}}\right)} A_{m, l}^{\chi} Z_{l}(z) \mathrm{e}^{\mathrm{i} m \theta}+\Phi_{\mathrm{p}, \chi}^{\mathrm{in}},
$$

where $A_{m, l}^{\chi}$ is the unknown coefficients to be determined; $\Phi_{\mathrm{p}, \chi}^{\mathrm{in}}$ is a particular solution,

$$
\begin{gathered}
\Phi_{\mathrm{p}, \chi}^{\mathrm{in}}= \begin{cases}0, & \chi=\mathrm{S} \\
-\frac{\mathrm{i}}{\rho \omega}, & \chi=\mathrm{R}\end{cases} \\
\tilde{I}_{m}\left(k_{l} r\right)= \begin{cases}J_{m}\left(k_{l} r\right), & l=0 \\
I_{m}\left(k_{l} r\right), & l \neq 0\end{cases}
\end{gathered}
$$

in which $J_{m}$ and $I_{\mathrm{m}}$ denote the Bessel function and the modified Bessel function of the first kind, respectively. $k_{0}$ is the wave number and $k_{l}(l>0)$ is the eigenvalue given by $[37,38]$,

$$
\omega^{2}=-k_{l} g \tan \left(k_{l} h\right), \quad l=1,2,3, \ldots
$$




$$
Z_{0}(z)=N_{0}^{-0.5} \cosh \left[k_{0}(z+h)\right] ; Z_{l}(z)=N_{l}^{-0.5} \cos \left[k_{l}(z+h)\right]
$$

229

230

231

233

234

where

$$
N_{0}=\frac{1}{2}\left[1+\frac{\sinh \left(2 k_{0} h\right)}{2 k_{0} h}\right], N_{l}=\frac{1}{2}\left[1+\frac{\sin \left(2 k_{l} h\right)}{2 k_{l} h}\right]
$$

II, ring region

$$
\Phi_{\chi}^{\mathrm{ring}}(r, \theta, z)=\sum_{m=0}^{\infty}\left[F_{m, 0}^{\chi}(r)+\sum_{l=1}^{\infty}\left(C_{m, l}^{\chi} \frac{I_{m}\left(\beta_{l} r\right)}{I_{m}\left(\beta_{l} R\right)}+D_{m, l}^{\chi} \frac{K_{m}\left(\beta_{l} r\right)}{K_{m}\left(\beta_{l} R\right)}\right) \cos \left[\beta_{l}(z+h)\right]\right] \cos m \theta
$$

where

$$
F_{m, 0}^{\chi}(r)=\left\{\begin{array}{ll}
C_{m, 0}^{\chi}+D_{m, 0}^{\chi}\left[1+\ln \left(\frac{r}{R}\right)\right], & m=0 \\
C_{m, 0}^{\chi}\left(\frac{r}{R}\right)^{|m|}+D_{m, 0}^{\chi}\left(\frac{r}{R}\right)^{-|m|}, & m \neq 0
\end{array},\right.
$$

in which $C_{m, l}^{\chi}$ and $D_{m, l}^{\chi}$ are the coefficients to be solved; $K_{m}$ is the modified Bessel function of the second kind; $\quad \beta_{l}$ is the $l$-th eigenvalue which is given by

$$
\beta_{l}=\frac{l \pi}{h-d}, l=0,1,2,3, \ldots
$$

III, outside region

$$
\Phi_{\chi}^{\text {out }}(r, \theta, z)=\sum_{m=0}^{\infty} \sum_{l=0}^{\infty} E_{m, l}^{\chi} \frac{\tilde{K}_{m}\left(k_{l} r\right)}{\tilde{K}_{m}\left(k_{l} R\right)} \cos (m \theta) Z_{l}(z)+\Phi_{\mathrm{p}, \chi}^{\text {out }},
$$

in which $E_{m, l}^{\chi}$ is the unknown coefficients to be determined; and $\Phi_{\mathrm{p}, \chi}^{\text {out }}$ is a particular solution,

$$
\Phi_{\mathrm{p}, \chi}^{\text {out }}=\left\{\begin{array}{ll}
\Phi_{1}, & \chi=\mathrm{S} \\
0, & \chi=\mathrm{R}
\end{array},\right.
$$

where, following Zheng and Zhang [37],

$$
\Phi_{\mathrm{I}}(r, \theta, z)=-\frac{2 \mathrm{i} g A}{\omega} \frac{Z_{0}(z)}{Z_{0}(0)} \sum_{m=0}^{\infty} \varepsilon_{m}(-\mathrm{i})^{m} J_{m}\left(k_{0} r\right) \cos m \beta \cos m \theta
$$

in which $\varepsilon_{m}=1$ for $m=0$, whereas $\varepsilon_{m}=2$ for $m \neq 0$.

$$
\tilde{K}_{m}\left(k_{l} r\right)=\left\{\begin{array}{ll}
H_{m}\left(k_{l} r\right), & l=0 \\
K_{m}\left(k_{l} r\right), & l \neq 0
\end{array},\right.
$$

where $H_{m}$ denotes the Hankel function of the first kind.

\subsection{Method of computation for unknown coefficients}

The scattering and radiated spatial potentials as expressed in Sections 3.1 satisfy all the 
boundary conditions shown in Eqs. (7) (12) already. Additionally, the boundary conditions at $r=R$ and $r=R_{\mathrm{i}}$, i.e., Eqs. (13) (15), together with the pressure and velocity continuity conditions on the interfaces of two adjacent regions should be satisfied as well, which can be used to determine the unknown coefficients in $\Phi_{\chi}$. These continuity conditions for $\Phi_{\chi}$ are given as follows:

1) Continuity of normal velocity at the boundary $r=R_{\mathrm{i}}$ :

$$
\frac{\partial \Phi_{\chi}^{\text {in }}}{\partial r}=\left\{\begin{array}{l}
0,-d<z<0, r=R_{\mathrm{i}}, 0 \leq \theta \leq \pi ; \text { and }-h<z<0, r=R_{\mathrm{i}}, \pi \leq \theta \leq 2 \pi \\
\frac{\partial \Phi_{\chi}^{\text {ring }}}{\partial r}, \quad-h<z<-d, r=R_{\mathrm{i}}, 0 \leq \theta \leq \pi
\end{array}\right.
$$

2) Continuity of normal velocity at the boundary $r=R$ :

$$
\frac{\partial \Phi_{\chi}^{\text {out }}}{\partial r}=\left\{\begin{array}{l}
0, \quad-d<z<0, r=R, 0 \leq \theta \leq \pi \\
\frac{\partial \Phi_{\chi}^{\text {ring }}}{\partial r}, \quad-h<z<-d, r=R, 0 \leq \theta \leq \pi
\end{array}\right.
$$

3) Continuity of pressure at the boundary $r=R_{\mathrm{i}}$ :

$$
\Phi_{\chi}^{\text {ring }}=\Phi_{\chi}^{\text {in }}, \quad-h<z<-d, r=R_{\mathrm{i}}, 0 \leq \theta \leq \pi
$$

4) Continuity of pressure at the boundary $r=R$ :

$$
\Phi_{\chi}^{\text {out }}=\Phi_{\chi}^{\text {ring }}, \quad-h<z<-d, r=R, 0 \leq \theta \leq \pi
$$

After inserting the expressions of $\Phi_{\chi}$ as given in Section 3.1 into these continuity conditions, i.e., Eqs.(29) (32), and making use of orthogonality of trigonometric functions and eigen-functions, the unknown coefficients in $\Phi_{\chi}$ can be determined. For convenience, details of the derivations are given in Appendix A.

\section{Excitation volume flow and hydrodynamic coefficients}

\subsection{Excitation volume flow}

Once the unknown coefficients are determined, the excitation volume flow $Q_{\mathrm{e}}$ as given in Eq. (3) can be easily calculated by:

$$
Q_{\mathrm{e}}=\frac{2 \pi \omega^{2} R_{\mathrm{i}}}{g}\left(-\frac{A_{0,0}^{\mathrm{D}}}{k_{0}^{2}} Z_{0}(z)+\sum_{l=1}^{\infty} \frac{A_{0, l}^{\mathrm{D}}}{k_{l}^{2}} Z_{l}(0)\right) .
$$

\subsection{Hydrodynamic coefficients}

In a similar way, the hydrodynamic coefficients as given in Eq. (4) can be rewritten in terms of $A_{m, l}^{\mathrm{R}}$ as:

$$
-(c-\mathrm{i} a)=Q_{\mathrm{R}}=\frac{2 \pi \omega^{2} R_{\mathrm{i}}}{g}\left(-\frac{A_{0,0}^{\mathrm{R}}}{k_{0}^{2}} Z_{0}(z)+\sum_{l=1}^{\infty} \frac{A_{0, l}^{\mathrm{R}}}{k_{l}^{2}} Z_{l}(0)\right) .
$$

The method, as shown in Eq. (4) or Eq. (34), which is derived from the radiated volume flow inside the column is a straightforward way for calculating the hydrodynamic coefficient, $c$. Actually there are two indirect methods as well for evaluating $c$, the one expressed by far-field coefficients as 


$$
c=2 \rho \omega h \sum_{m=0}^{\infty} \frac{1}{\varepsilon_{m}} \frac{\left|E_{m, 0}^{\mathrm{R}}\right|^{2}}{\left|H_{m}\left(k_{0} R\right)\right|^{2}},
$$

which can be derived from Green's identity [36, 39]; and the other one derived from the excitation volume flow $Q_{\mathrm{e}}$ based on Haskind Relation [32, 39]:

$$
c=\frac{\omega Z_{0}(0)^{2}}{8 \pi \rho g^{2} h} \int_{0}^{\pi}\left|Q_{\mathrm{e}}(\beta)\right|^{2} \mathrm{~d} \beta .
$$

The comparison of the results of $c$ by using these two indirect methods as given in Eqs. (35) and (36) with that of the direct method, i.e., Eq. (4) or Eq. (34), can be adopted as an approach to validate the theoretical model.

\section{Model validation}

Martins-rivas and Mei [32] solved the hydrodynamic problems from a thin-walled (i.e., $R_{\mathrm{i}}=R$ ) OWC along a straight coast for $R / h=0.5, d / h=0.2$ subjected to regular waves propagating at different angles $\beta$ with different values of $k h$. The present theoretical model without the thin-wall restriction is adopted to re-simulate the same case, in which the inner radius is chosen as $R_{\mathrm{i}} / h=0.49$, i.e., $\left(R-R_{\mathrm{i}}\right) / R=0.02$, to represent the thin chamber wall.

To make a comparison with the published results, the method as adopted in Martins-rivas and Mei [32] for nondimensionalizing $Q_{\mathrm{e}}$ and hydrodynamic coefficients, $c$ and $a$, i.e., $\bar{Q}_{\mathrm{e}}=\omega Q_{\mathrm{e}} /(A R g), \quad(\bar{c}, \bar{a})=(c, a) \omega \rho / R$, is re-employed in the present section.

\subsection{Wave scattering problem}

The comparison between the present results of the free surface elevation pattern inside and outside the OWC for $k h=1.64$ and those of Martins-rivas and Mei [32] is given in Fig. 2. 

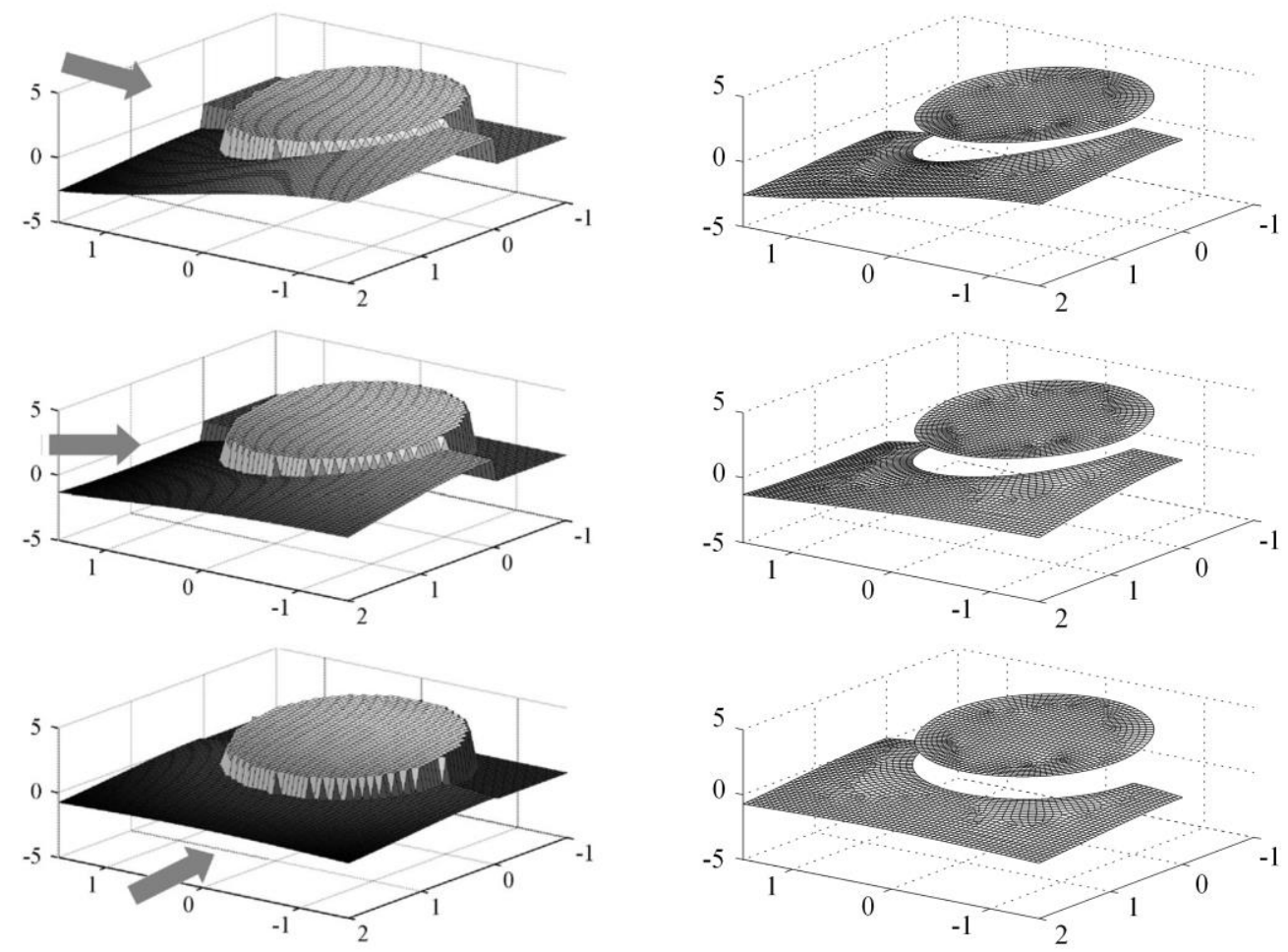

Fig. 2. Free surface elevation inside and outside the OWC for $R / h=0.5, d / h=0.2, k h=1.64, t=\pi / 2 \omega$. (left) results of Martins-rivas and Mei [32] for thin-walled $\mathrm{OWC}$, i.e., $R_{\mathrm{i}}=R$; (right) present results with $R_{\mathrm{i}} / h=0.49$. The incidence angles $\beta=0,0.25 \pi, 0.5 \pi$.

In addition, comparison of the excitation volume flow of the $\mathrm{OWC}$ as a function of incidence $\beta$ for $R / h=0.5, d / h=0.2, k h=3.170$ and 1.802 by using the present model with that of Martins-rivas and Mei [32] is illustrated in Fig. 3.

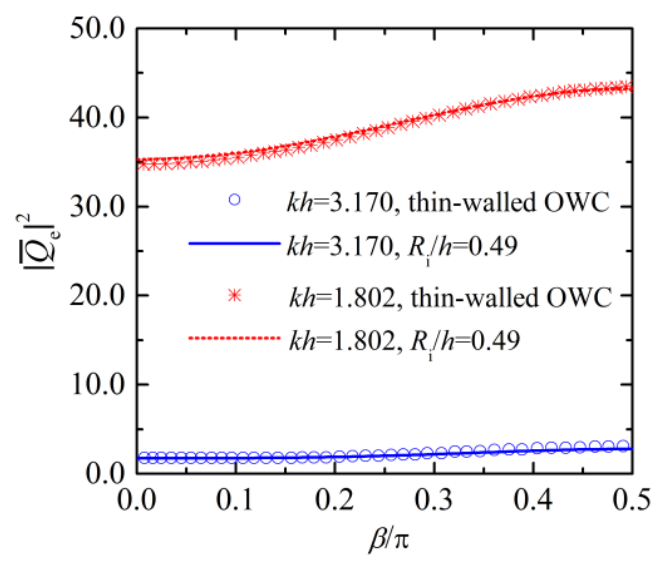

Fig. 3. Excitation volume flow of the OWC as a function of incidence $\beta$ for $R / h=0.5, d / h=0.2$. symbols: results from Martins-rivas and Mei [32] for thin-walled OWC, i.e., $R_{\mathrm{i}}=R$; lines: present results with $R_{\mathrm{i}} / h=0.49$.

The excellent agreement of the present results with those of Martins-rivas and Mei [32], as shown in Figs. 2 and 3, proves that the present theoretical model works pretty well in solving the scattering problem. 
Figure 4 illustrates the frequency response of the hydrodynamic coefficients of the OWC with $R / h=0.5, d / h=0.2$. It can be learnt that the present results of $c$ and $a$ with $R_{\mathrm{i}} / h=0.49$, i.e., $\left(R-R_{\mathrm{i}}\right) / R=0.02$, are in rather good agreement with those under thin-wall restriction [32]. What is more, results of $c$ for the case with $R_{\mathrm{i}} / h=0.4$ by using direct method (denoted as DM) and by adopting the other two indirect methods (denoted as FFC and HR, respectively) agree with each other pretty well, meaning the correctness of the present model in solving wave radiation problem.

(a)

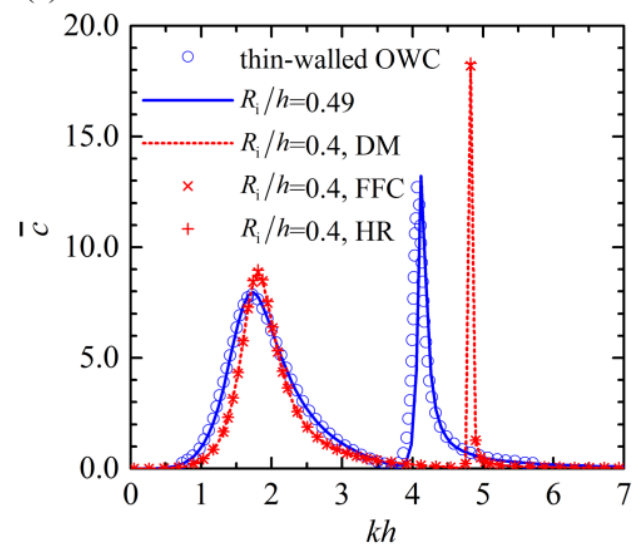

(b)

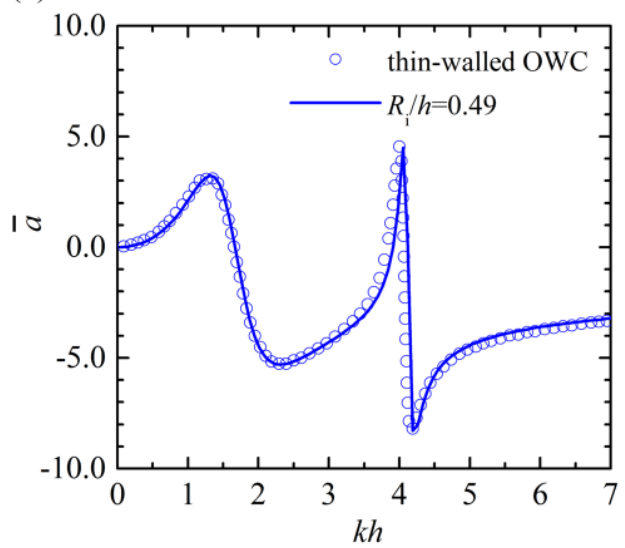

Fig. 4. Frequency response of radiation damping and added mass of the OWC with $R / h=0.5$, $d / h=0.2$ : (a) radiation damping; (b) added mass. Circles: results from Martins-rivas and Mei [32] for thin-walled OWC, i.e., $R_{\mathrm{i}}=R$; lines and crosses: present results.

Note there is an obvious difference of the frequency response of radiation damping by using $R_{\mathrm{i}} / h=0.4$ and $R_{\mathrm{i}} / h=0.49$, reflecting the significant effect of the OWC chamber's thickness on the hydrodynamic characteristics of the OWC along a vertical wall. Influence of the thickness on the performance of the OWC deserves more attention and such effect will be discussed in the next section.

\section{Results and discussion}

Hereinafter, following Lovas, Mei [33], the dimensionless coefficients of $Q_{\mathrm{e}}$ and hydrodynamic coefficients, $c$ and $a$, are redefined as follows:

$$
\bar{Q}_{\mathrm{e}}=\frac{\sqrt{g / h}}{A h g} Q_{\mathrm{e}} ;\left(\bar{c}, \bar{a}, \bar{c}_{\mathrm{PTO}}, \bar{a}_{\mathrm{PTO}}\right)=\frac{\rho \sqrt{g / h}}{h}\left(c, a, c_{\mathrm{PTO}}, a_{\mathrm{PTO}}\right),
$$

with which, Eq. (6) can be rewritten as

$$
\eta=\frac{k h g}{c_{\mathrm{g}} \sqrt{g / h}} \frac{\bar{c}_{\text {РTO }}\left|\bar{Q}_{\mathrm{e}}\right|^{2}}{\left[\left(\bar{c}+\bar{c}_{\mathrm{PTO}}\right)^{2}+\left(\bar{a}+\bar{a}_{\mathrm{PTO}}\right)^{2}\right]} .
$$

Following Martins-rivas and Mei [32] and Lovas, Mei [33], $a_{\text {PTO }}$ is calculated based on $\rho / \rho_{0}=1000, v=340 \mathrm{~m} / \mathrm{s}, h=10 \mathrm{~m}$, and $V_{0}=\pi R^{2} h$. The corresponding optimal PTO damping for a fixed OWC chamber (fixed $V_{0}$ ) is obtained by requiring $\partial P / \partial c_{\mathrm{PTO}}=0$,

$$
c_{\mathrm{PTO}}=\sqrt{c^{2}+\left(a+a_{\mathrm{PTO}}\right)^{2}} \text {. }
$$


Figure 5 presents the frequency responses of wave excitation volume flux, hydrodynamic coefficients, turbine parameter and wave power capture factor when the coast/breakwater integrated OWC suffers from different incident directions. For reasons of symmetry, only the results for $\beta=\pi / 6, \pi / 4, \pi / 3$ and $\pi / 2$ are presented. For comparison, the results for the same OWC in the open sea are also displayed [36]. Only one blue dash curve as plotted in each figure of Figs. $5 \mathrm{~b} \sim 5 \mathrm{~d}$ is used to represent the results of $\bar{c}, \bar{a}$ and $\bar{c}_{\mathrm{PTO}}$ for the coast/breakwater integrated OWC, respectively, since they are all independent of $\beta$.

For wave scattering problem of the isolated offshore OWC, in the computed range of $k h$, there is only one peak of $\left|\bar{Q}_{\mathrm{e}}\right|-k h$ curve at $k h \approx 2.44$ (see Fig. 5a). Whereas for the coast/breakwater integrated OWC, apart from the main peak of $\left|\bar{Q}_{\mathrm{e}}\right|-k h$ curve at $k h \approx 1.73$, a second sharp peak is also observed at a higher frequency, i.e., $k h \approx 4.82$. Such a feature can be identified from the view of natural modes in a closed cylinder in the radiation problem. For the isolated offshore OWC, the only peak of the $\bar{c}-k h$ curve occurs at $k h \approx 2.44$ (Fig. 5b), which corresponds to a piston-like motion, i.e., the so-called Helmholtz mode of oscillation. In a coast/breakwater integrated OWC, the Helmholtz mode cannot exist alone because of the asymmetry of the opening; another mode, i.e., the sloshing mode, is excited [32], and dominates the water motion inside the OWC chamber at $k h \approx 4.82$. As shown in Fig. $5 \mathrm{c}$, the $\bar{a}-k h$ performs like a $\mathrm{N}$ letter shaped and a two-N letter shaped curves for an isolated offshore OWC and the integrated case, respectively, and the sign of $\bar{a}$ changes rapidly at the $k h$ where the peak of the $\bar{c}-k h$ curve occurs. These values of $k h$ can be called the natural frequencies of the OWC in the absence of the PTO. The spiky behaviour of $\bar{a}$ around these natural frequencies is connected to the peak of the $\bar{c}-k h$ curve. Note that the chamber coefficient $\left(-\bar{a}_{\mathrm{PTO}}\right)$ is also plotted in Fig. $5 \mathrm{~d}$ as a gray solid curve, which intersects the $\bar{a}-k h$ curve at $k h \approx(2.48,4.18)$ and $(1.86,2.90,4.90)$ for different cases. The $k h$-values where these intersections occur correspond the resonant frequencies of the OWC with the PTO system. For wave conditions corresponding to these resonant frequencies, $\bar{a}$ and $\bar{a}_{\text {PTO }}$ cancel each other, and it can be readily known from Eq. (2) that the air pressure inside the OWC chamber is in phase with the excitation volume flux. The frequency response of the optimal turbine parameter $\bar{c}_{\text {PTO }}$ is illustrated in Fig. 5d, in which $\bar{c}_{\text {PTO }}=\bar{c}$ is satisfied at the resonant frequencies. Compared with the single offshore OWC, the variation of $\bar{c}_{\text {PTO }}$ for the coast/breakwater integrated OWC is less marked and less abrupt (except for $k h \approx 4.9$ ), which means that it may be easier to achieve in practice.

Finally, $\eta$ as a function of $k h$ for different values of the incident wave angle $\beta$ is given in Fig. 5e. It is apparent that $\eta$ for the isolated offshore OWC reaches the theoretical maximum value (i.e., $1.0)$ at these two resonant frequencies. Peaks of the $\eta-k h$ curve for the integrated case are also observed at the corresponding resonant frequencies. Thanks to the wave reflection from the coast/breakwater, the value of $\eta$ for the coast/breakwater integrated OWC at $1.6<k h<3.1$ can be around twice as large as the theoretical maximum for the offshore case. Note that there is a 
frequency between the second and the third resonant frequencies where no power can be extracted.
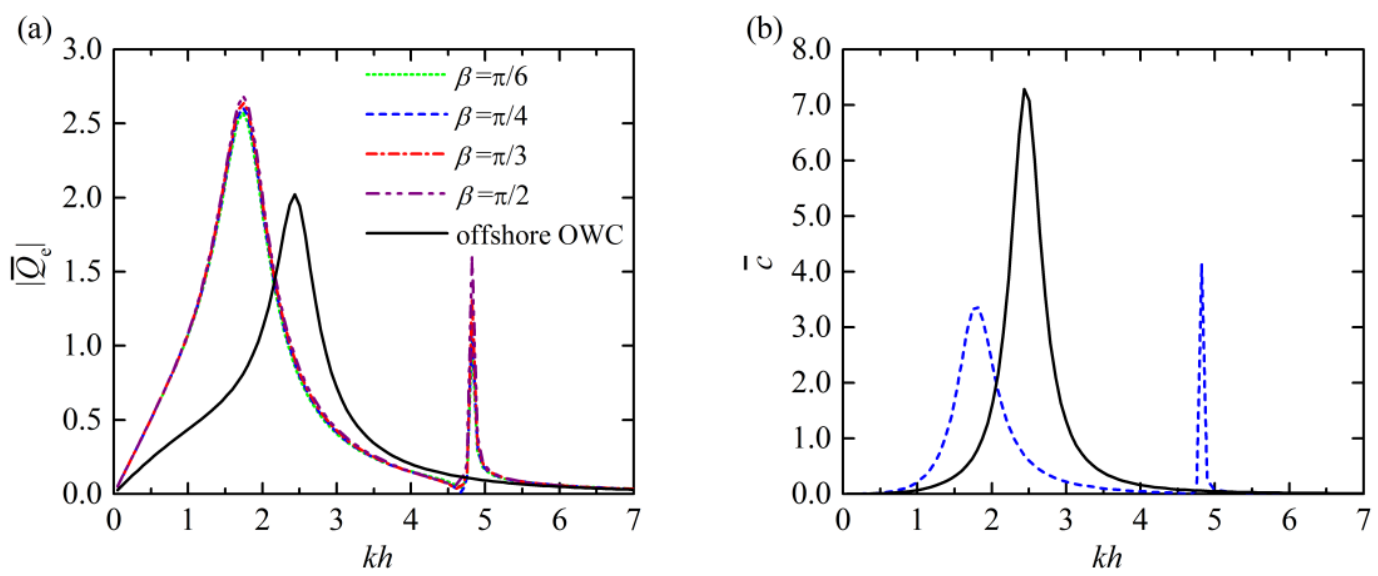

380
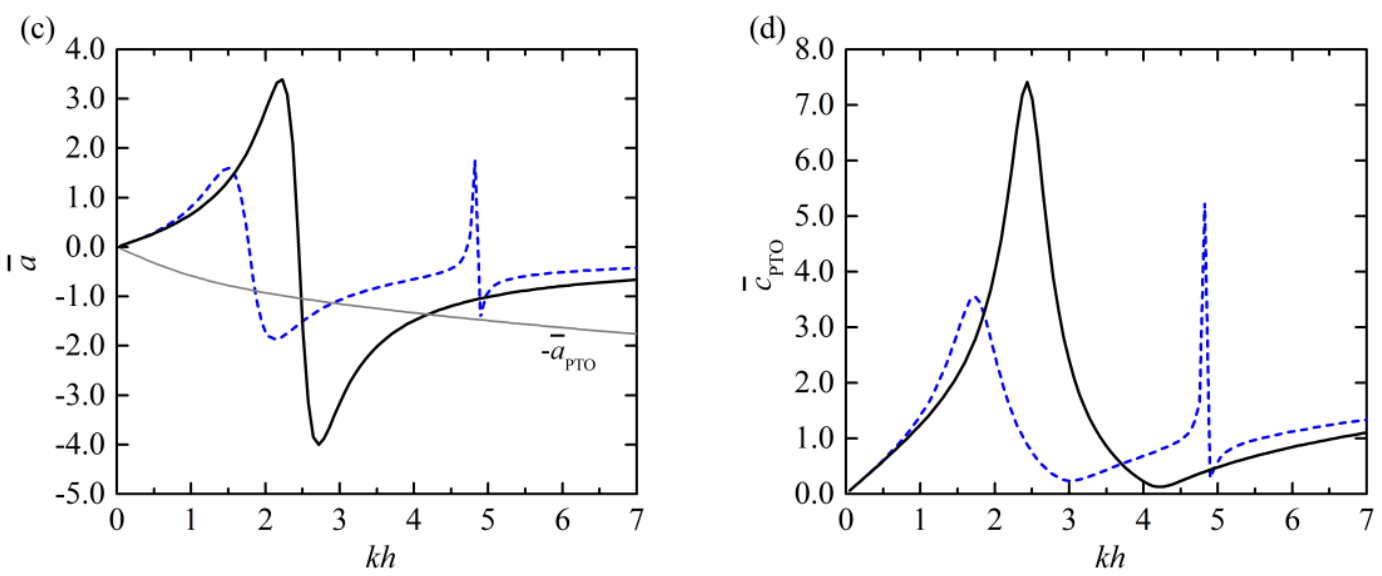

381

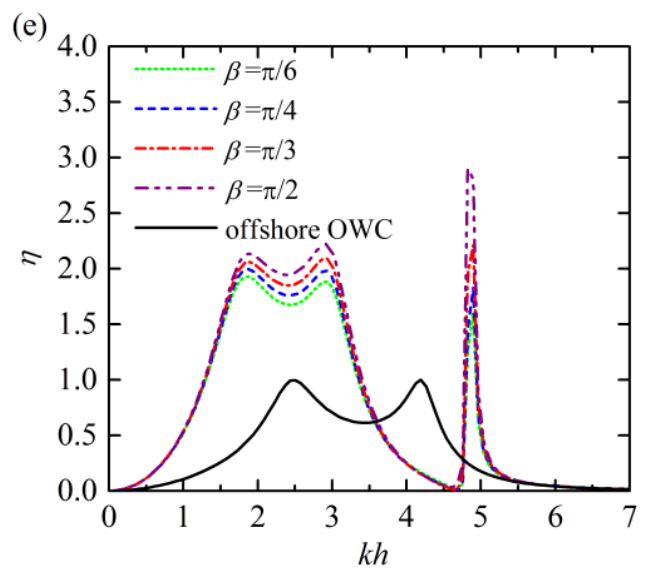

382

383

384

385

Fig. 5. Comparison for different incident wave directions, $\beta$. (a) wave excitation volume flux $\left|\bar{Q}_{\mathrm{e}}\right|$; (b) radiation damping $\bar{c}$; (c) added mass $\bar{a}$ and chamber coefficient $-\bar{a}_{\mathrm{PTO}} \quad$ (gray solid line); (d) turbine parameter $\bar{c}_{\text {РTO }}$; (e) wave power capture factor $\eta$. In every case, $R / h=0.5$, $\left(R-R_{\mathrm{i}}\right) / h=0.1, d / h=0.2$. Black solid line: isolated offshore OWC.

The effect of the incident wave direction $\beta$ on $\left|\bar{Q}_{\mathrm{e}}\right|$ is not obvious (Fig. 5a). As $\beta$ increases from $\pi / 6$ to $\pi / 2$, this dependence is slightly visible at the natural frequencies. As a comparison, a 
401

402
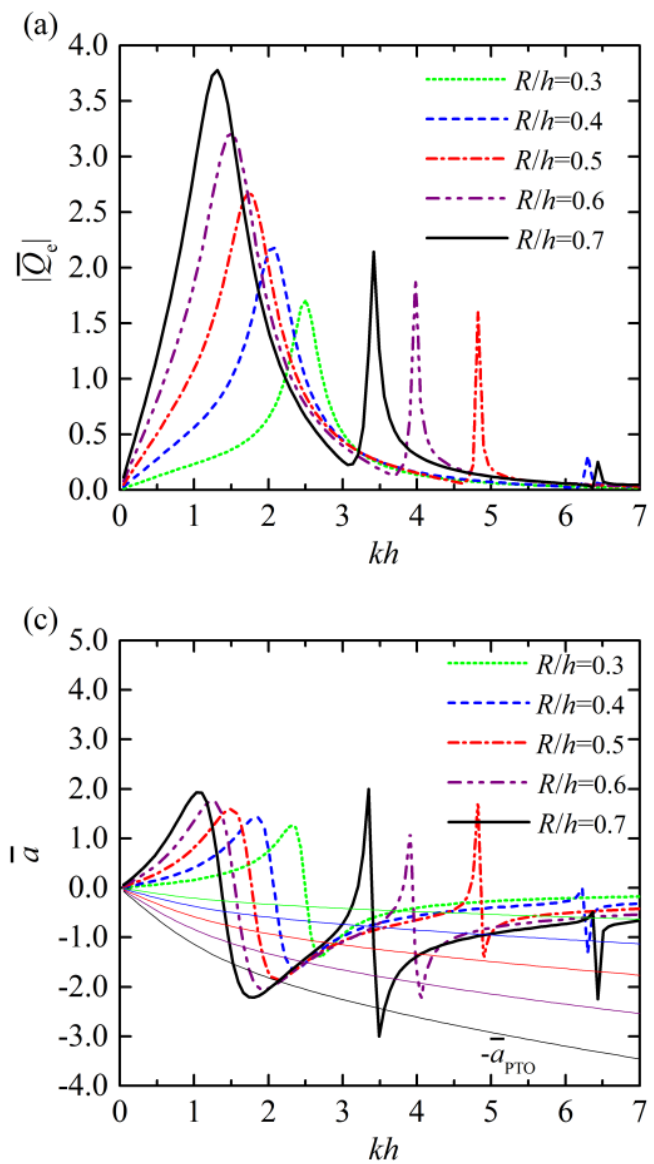
with $\beta=\pi / 2$.

\subsection{Radius of the OWC chamber}

significant influence of $\beta$ on $\eta$ can be observed for $1.6<k h<3.1$ and $4.9<k h<5.0$ (Fig. 5e), where the more perpendicular the incident wave direction relative to the coast/breakwater, the more wave power that can be captured. Hereinafter, the effects of the other parameters will all be examined

The coast/breakwater integrated OWCs with $R / h=0.3 \sim 0.7$ are selected as five cases to investigate the effect of the radius of the chamber on the performance of the OWC (Fig. 6). As $R / h$ increases, the highest peak of the $\left|\bar{Q}_{\mathrm{e}}\right|-k h$ curve (Fig. 6a) shifts toward a lower frequency and gains intensity. Similar changes affect $\bar{c}, \bar{a}$ and $\bar{c}_{\mathrm{PTO}}$ (Figs. 6b 6d). As $R / h$ increases, more natural frequencies can be observed in the computed range of $k h$. For $R / h=0.3$, there is only one natural frequency in the range of $k h$ plotted, whereas for $R / h=0.4,0.5$ and 0.6 , there are two. For $R / h=0.7$, three natural frequencies are readily observable.
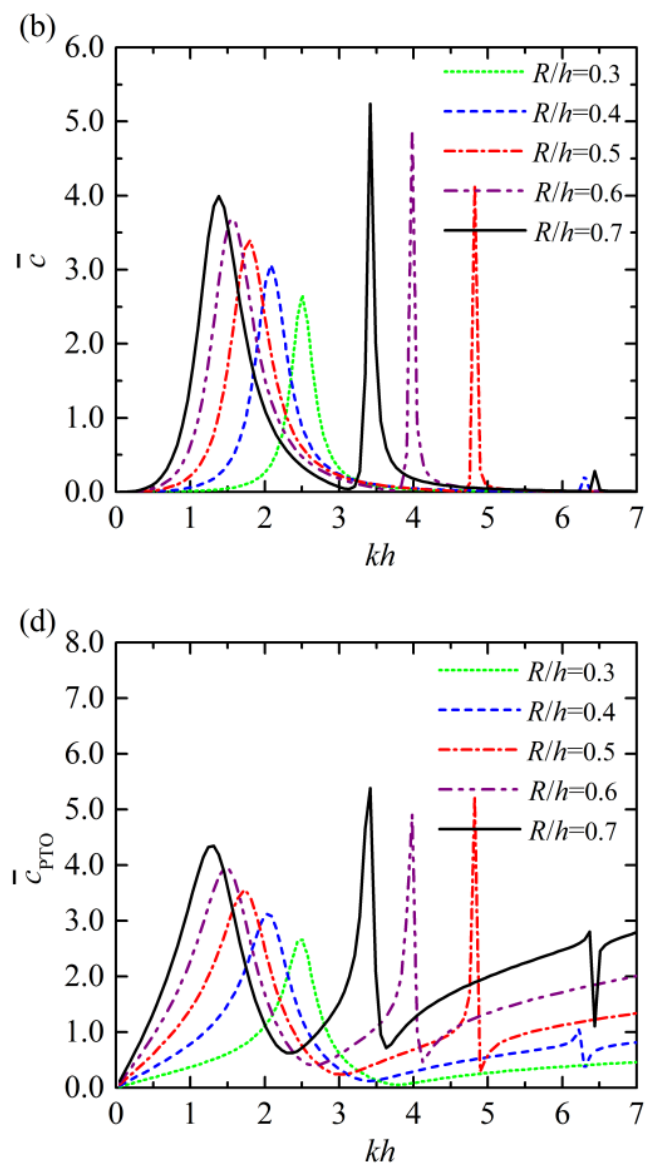


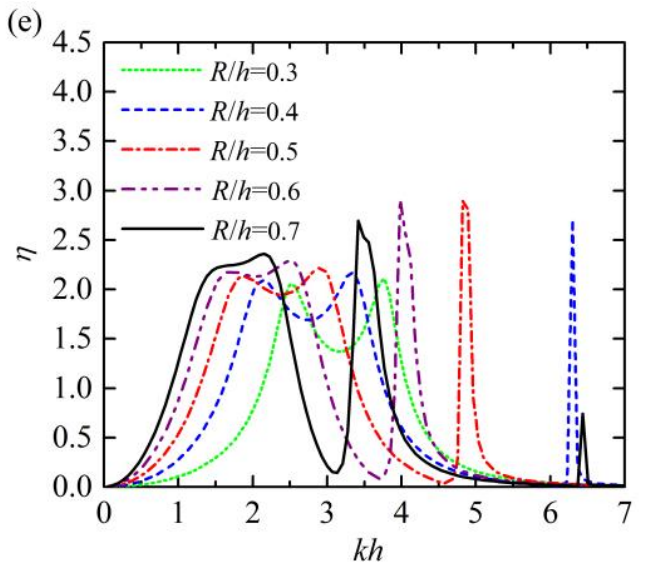

404

Fig. 6. Comparison for different radii of the OWC chamber, $R / h$. (a) wave excitation volume flux $\left|\bar{Q}_{\mathrm{e}}\right|$; (b) radiation damping $\bar{c}$; (c) added mass $\bar{a}$ and chamber coefficient $-\bar{a}_{\text {PTо }}$ (thin solid lines, each of which corresponds to the line of $\bar{a}$ plotted in the same color); (d) turbine parameter $\bar{c}_{\mathrm{PTO}} ;(\mathrm{e})$ wave power capture factor $\eta$. In every case, $\left(R-R_{\mathrm{i}}\right) / h=0.1, d / h=0.2, \beta=\pi / 2$.

Given that the chamber volume $V_{0}\left(V_{0}=\pi R^{2} h\right)$ is dependent on $R$, there are also five $\left(-\bar{a}_{\text {PTO }}\right)-k h$ curves plotted in Fig. $6 \mathrm{c}$ corresponding to different values of $R / h$. For $R / h=0.3$, there are only two resonant frequencies in the computed range of $k h$. For the other cases, e.g., $R / h=0.6$, there could be four resonant frequencies in the same range of $k h$. As plotted in Fig. $6 \mathrm{~d}$, the larger the $R / h$, the higher and more abrupt the variation of $\bar{c}_{\text {PTO }}$. The plot of $\eta$ (Fig. 6e) shows that when $k h$ is between the first two resonant frequencies, as $R / h$ increases, the $\eta-k h$ curve turns higher and flatter, and shifts toward lower frequencies. Here, $\Delta k h$ is adopted to denote the difference between the first two resonant frequencies, and $\eta_{1}$ and $\eta_{2}$ are employed to represent the $\eta$-values corresponding to the first two resonant frequencies, respectively. Figure 7 presents $\eta_{1}, \eta_{2}$ and $\Delta k h$ as three functions of $R / h$. It is clear that, as $R / h$ increases from 0.3 to 0.7 , both $\eta_{1}$ and $\eta_{2}$ increase in a linear way approximately, whereas $\Delta k h$ decreases dramatically from 1.19 to 0.56 . Ideally the $R / h$ ratio should be selected to achieve the balance between the peak value of $\eta$ and its bandwidth, so that the OWC can capture the most power for a specified range of wave conditions.

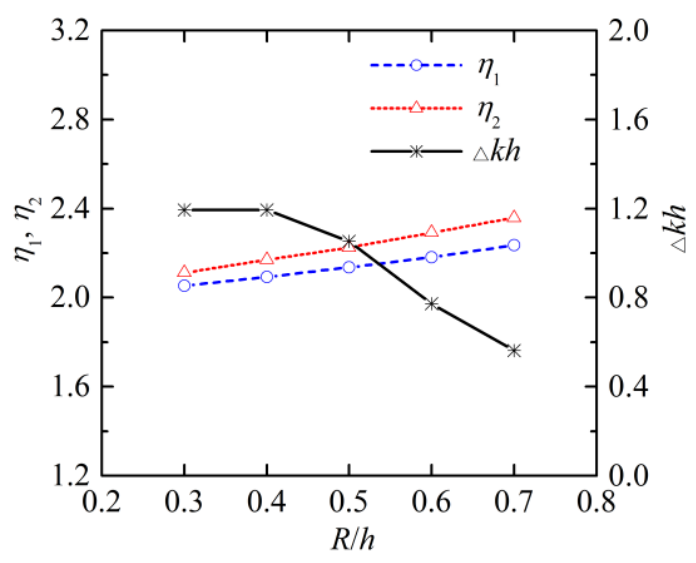

Fig. 7. Variation of $\eta_{1}, \eta_{2}$ and $\Delta k h$ with $R / h$ for $\left(R-R_{\mathrm{i}}\right) / h=0.1, d / h=0.2, \beta=\pi / 2$ 


\subsection{Thickness of the OWC chamber}

Figure 8 presents the results of the OWC chamber with $\left(R-R_{\mathrm{i}}\right) / h=0.05,0.1,0.15$ and 0.2 , and the other parameters fixed at $R / h=0.5, d / h=0.2, \beta=\pi / 2$. For comparison, some results of the thin-wall case, i.e., $\left(R-R_{\mathrm{i}}\right) / h=0$, which were previously displayed by Martins-rivas and Mei [47] and Lovas et al. [48], are replotted in Fig. 8 as well. As $\left(R-R_{\mathrm{i}}\right) / h$ increases, the inner radius of the chamber decreases, and the highest peak of $\left|\bar{Q}_{\mathrm{e}}\right|-k h$ curve loses intensity and moves toward higher frequency as expected (see Fig. 8a). Meanwhile, the main peak of $\bar{c}$ shifts toward higher frequency and turns higher and narrower. Similar changes are also found for $\bar{a}$ and $\bar{c}_{\text {PTO }}$ as given in Figs. $8 \mathrm{c}$ and $8 \mathrm{~d}$. It should be noted from Fig. $8 \mathrm{c}$ that with the increase of $\left(R-R_{\mathrm{i}}\right) / h$, the first two intersection points of $\bar{a}$ and $-\bar{a}_{\text {PTO }}$ get closer and closer to each other horizontally, hence the frequency band of $\eta-k h$ as plotted in Fig. 8e turns narrower and narrower, whereas the frequency position of the middle of the band remains almost the same. In addition, the peaks of $\eta$ corresponding to the first two resonant frequencies are lower as $\left(R-R_{\mathrm{i}}\right) / h$ increases. Therefore, generally, the thickness of the OWC chamber should be as small as possible, so that larger and broader main peaks can be achieved and more wave power absorbed. Needless to say, the minimum thickness will be dictated in practice by structural considerations.
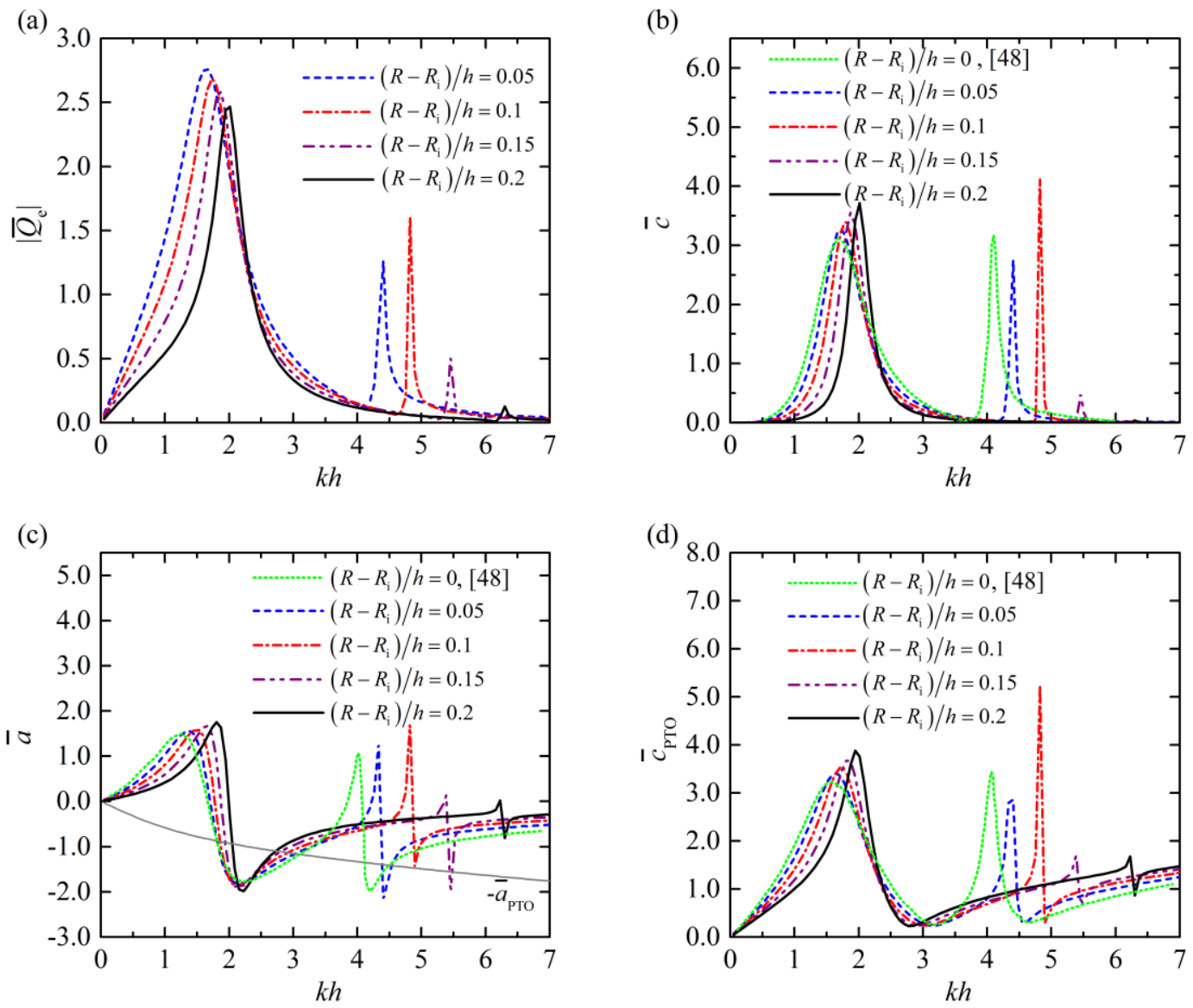


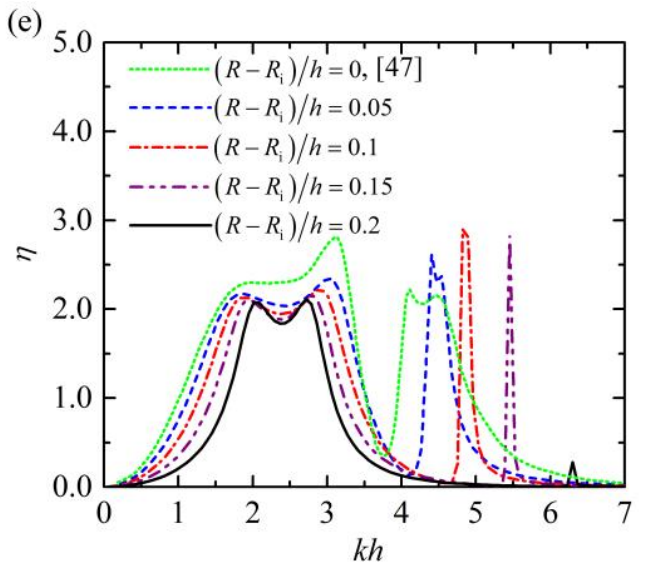

443

Fig. 8. Comparison for different thicknesses of the OWC chamber wall, $\left(R-R_{\mathrm{i}}\right) / h$. (a) wave excitation volume flux $\left|\bar{Q}_{\mathrm{e}}\right|$; (b) radiation damping $\bar{c} ;$ (c) added mass $\bar{a}$ and chamber coefficient $-\bar{a}_{\mathrm{PTO}}$ (gray solid line); (d) turbine parameter $\bar{c}_{\mathrm{PTO}} ;$ (e) wave power capture factor $\eta$. In every case, $R / h=0.5, d / h=0.2, \beta=\pi / 2$.

\subsection{Submergence of the OWC chamber}

Figure 9 compares the results for the coast/breakwater integrated OWC with different submergence of the chamber, i.e., $d / h=0.1,0.15,0.2,0.25$ and 0.3 , with $R / h=0.5,\left(R-R_{\mathrm{i}}\right) / h=0.1$, $\beta=\pi / 2$. The results of $\left|\bar{Q}_{\mathrm{e}}\right|$ (Fig. 9a) show that with the increase of $d / h$, the highest peak of $\left|\bar{Q}_{\mathrm{e}}\right|$ turns higher and narrower, and shifts toward lower frequencies. Similar changes apply to $\bar{c}$ and $\bar{a}$ (Figs. 9b and 9c). This is reasonable, for the radiation loss becomes weaker. All the resonant frequencies in the computed range of $k h$ reduce as $d / h$ increases. The larger the $d / h$ ratio, the higher and more abrupt the variation of the corresponding $\bar{c}_{\text {PTO }}$ with $k h$ (Fig. 9d), which may be more difficult to achieve in practice. The plot of $\eta$ in Fig. 9e shows that, due to the change in the resonant frequencies, the peaks of $\eta$ are shifted toward lower frequencies as well with the increase in $d / h$. Meanwhile, both $\eta_{1}$ and $\eta_{2}$ are found to decrease slightly, and the main bandwidth in terms of $\Delta k h$ also decreases. The $\eta$ corresponding to the $k h$ between the first two resonant frequencies decreased more dramatically than $\eta_{1}$ and $\eta_{2}$. It might be concluded that a better result can be obtained by using a smaller value of $d / h$. 
(a)

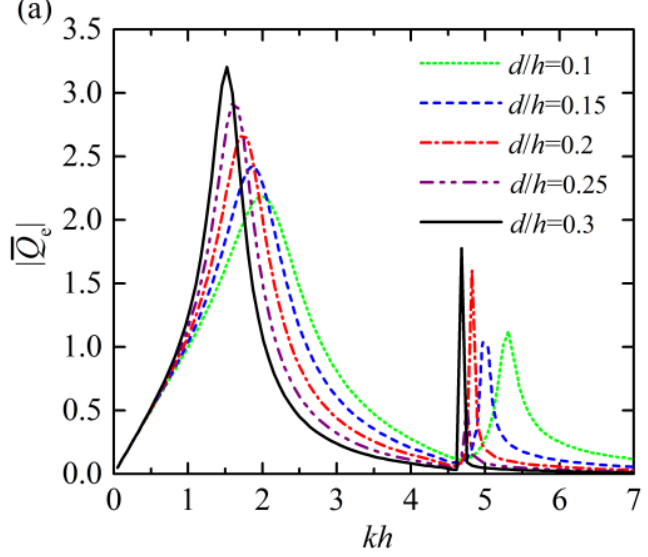

463

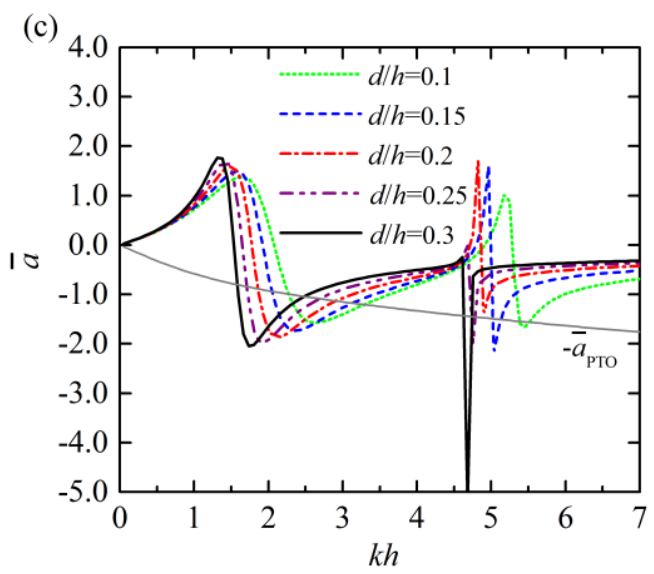

(b)

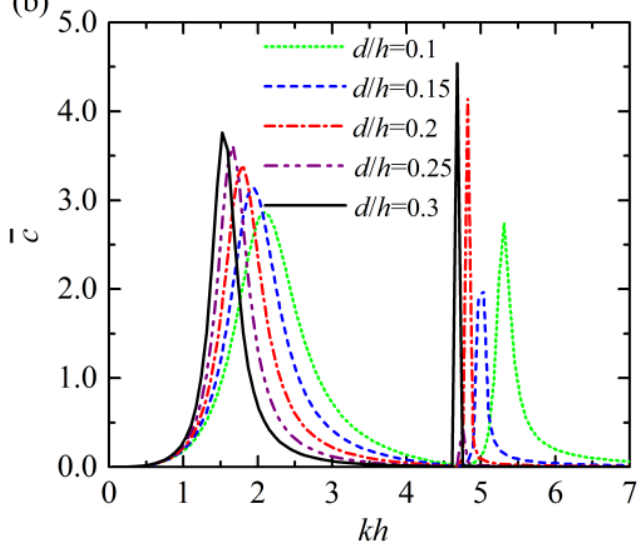

(d)

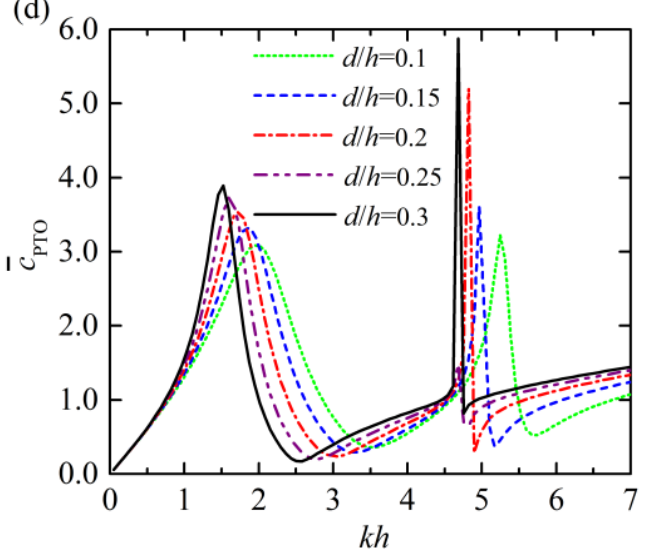

(e)

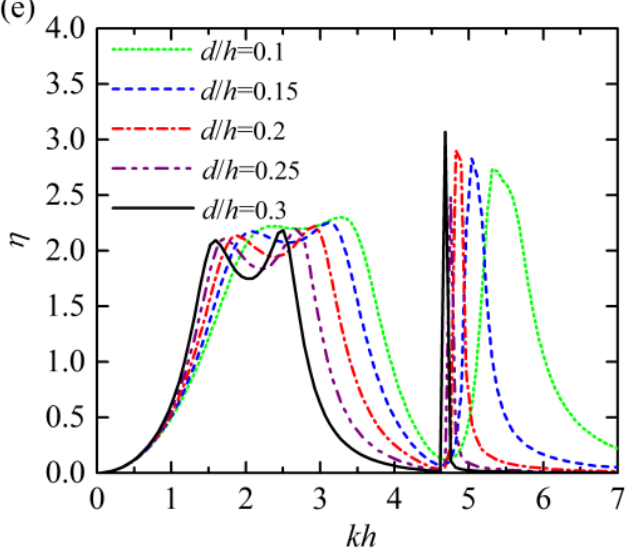

Fig. 9. Comparison for different submergence of the OWC chamber, $d / h$. (a) wave excitation volume flux $\left|\bar{Q}_{\mathrm{e}}\right|$; (b) radiation damping $\bar{c}$; (c) added mass $\bar{a}$ and chamber coefficient $-\bar{a}_{\mathrm{PTO}}$ (gray solid line); (d) turbine parameter $\bar{c}_{\text {PTO }}$; (e) wave power capture factor $\eta$. In every case,

$$
R / h=0.5,\left(R-R_{\mathrm{i}}\right) / h=0.1, \beta=\pi / 2 .
$$

Note that $d / h$ cannot be too small in practice, otherwise the opening might not be continuously submerged, especially when the OWC is subjected to incident waves with a large amplitude, to a large tidal range, or both. 


\section{Conclusions}

In this paper a theoretical model based on linear potential flow theory is proposed to study the performance of an OWC along a vertical coast/breakwater without the thin-wall restriction of previous works. The water domain is divided into three regions, i.e., the interior region enclosed by the OWC chamber, the half-ring shaped region beneath the OWC chamber and the exterior region in front of the coast/breakwater extending towards infinite distance horizontally. Subjected to small amplitude incident regular waves, wave-structure interaction is decomposed into wave scattering and wave radiation problems. In order to determine the unknown coefficients of the scattering and radiated potentials in these three regions, the eigen-function matching method is employed. The wave power extraction of the OWC with linear PTO system is then evaluated in the frequency domain.

The influence of the vertical coast/breakwater is briefly discussed by comparing the performance of the integrated OWC with that of a similar isolated OWC deployed in the open sea. Finally, the effects of the radius, thickness and submergence of the chamber on the performance of the OWC along a coast/breakwater are investigated by means of the theoretical model. The following conclusions may be drawn.

The value of $\eta$ for the coast/breakwater integrated OWC at specified ranges of $k h$ can be around twice as large as the theoretical maximum of $\eta$ for the offshore case due to the wave reflection from the coast/breakwater. The more perpendicular the incident wave direction relative to the coast/breakwater, the more wave power that can be captured by the OWC.

As the $R / h$ ratio increases, more natural and resonant frequencies can be observed in the computed range of $k h$. The main peaks of $\eta$ shift toward lower frequencies and the peak values increase nearly linearly with $R / h$, whereas the bandwidth reduces drastically.

The smaller the $\left(R-R_{\mathrm{i}}\right) / h$ ratio, the larger and broader the main peaks of $\eta$, i.e., more wave power absorbed, and the frequency position of the middle of the band remains almost the same. Needless to say, an appropriate thickness, rather than zero thickness of the OWC chamber, will be dictated in practice by overall considerations, including not only wave power extraction but also structural survivability.

With the increase of $d / h$, the peaks of $\eta$ are shifted toward lower frequencies. Meanwhile, both $\eta_{1}$ and $\eta_{2}$ are found to decrease slightly, and the main bandwidth in terms of $\Delta k h$ is reduced.

To capture wave power on a large scale, it is expected that multiple OWCs along a coast/breakwater will be required. It is possible to extend the present theoretical model to multiple OWCs, as will be reported elsewhere.

\section{Acknowledgements}

The research was supported by National Natural Science Foundation of China (51679124, 51479092) and Intelligent Community Energy (ICE), INTERREG V FCE, European Commission (Contract No. 5025).

Appendix A. Integral equations of the scattering and radiation problems

After inserting Eqs. (16) and (22) into Eq.(29), multiplying both sides by $Z_{\zeta}(z) \mathrm{e}^{-i \tau \theta}$ and integrating for $z \in[-h, 0]$ and $\theta \in[0,2 \pi]$, for any pair of integer $(\tau, \zeta)$, it can be obtained that 
$5142 \pi h A_{\tau, \zeta}^{\chi}=\sum_{l=0}^{\infty}\left[\frac{\pi}{\varepsilon_{|\tau|}}\left(X_{|\tau|, \mid}^{(1)} C_{|\tau|, l}^{\chi}+Y_{|\tau|, \mid}^{(1)} D_{|\tau|, l}^{\chi}\right)+\mathrm{i} \sum_{\substack{m=0 \\ m \neq|\tau|}}^{\infty} \frac{\tau\left[(-1)^{\tau-m}-1\right]}{\tau^{2}-m^{2}}\left(X_{m, l}^{(1)} C_{m, l}^{\chi}+Y_{m, l}^{(1)} D_{m, l}^{\chi}\right)\right] L_{l, \zeta}$,

515

516

517

where

(A.1)

where

518

$$
X_{\tau, \zeta}^{(1)}=\left\{\begin{array}{l}
\frac{\tau}{R}\left(\frac{R_{\mathrm{i}}}{R}\right)^{\tau-1}, \quad \zeta=0 \\
\frac{\beta_{\zeta} I_{\tau}^{\prime}\left(\beta_{\zeta} R_{\mathrm{i}}\right)}{I_{\tau}\left(\beta_{\zeta} R\right)}, \quad \zeta \neq 0
\end{array} \quad ; \quad Y_{\tau, \zeta}^{(1)}= \begin{cases}\frac{1}{R_{\mathrm{i}}}, & \zeta=0, \tau=0 \\
-\frac{\tau}{R}\left(\frac{R}{R_{\mathrm{i}}}\right)^{\tau+1}, & \zeta=0, \tau \neq 0 . \\
\frac{\beta_{\zeta} K_{\tau}^{\prime}\left(\beta_{\zeta} R_{\mathrm{i}}\right)}{K_{\tau}\left(\beta_{\zeta} R\right)}, & \zeta \neq 0\end{cases}\right.
$$

$$
L_{l, \zeta}=\int_{-h}^{-d} \cos \left[\beta_{l}(z+h)\right] Z_{\zeta}(z) \mathrm{d} z
$$

$$
= \begin{cases}\frac{(-1)^{l}(h-d)^{2} k_{0} Z_{0}(0) \sinh \left[k_{0}(h-d)\right]}{\left[(h-d)^{2} k_{0}^{2}+l^{2} \pi^{2}\right] \cosh \left(k_{0} h\right)}, & \zeta=0 . \\ \frac{(-1)^{l}(h-d)^{2} k_{\zeta} Z_{\zeta}(0) \sin \left[k_{\zeta}(h-d)\right]}{\left[(h-d)^{2} k_{\zeta}^{2}-l^{2} \pi^{2}\right] \cos \left(k_{\zeta} h\right)}, & \zeta \neq 0 .\end{cases}
$$

519

520

521

After inserting Eqs. (22) and (25) into Eq.(30), multiplying both sides by $Z_{\zeta}(z) \cos (\tau \theta)$ and integrating for $z \in[-h, 0]$ and $\theta \in[0, \pi]$, for any pair of integer $(\tau, \zeta)$, we have

$$
\sum_{l=0}^{\infty}\left(X_{\tau, l}^{(2)} C_{\tau, l}^{\chi}+Y_{\tau, l}^{(2)} D_{\tau, l}^{\chi}\right) L_{l, \zeta}-h Z_{\tau, \zeta}^{(2)} E_{\tau, \zeta}^{\chi}=f_{2}^{\chi}
$$

522

523

in which

524

$$
\begin{gathered}
f_{2}^{\chi}= \begin{cases}-\frac{2 \delta_{\zeta, 0} \mathrm{i} g A k_{0} h}{\omega Z_{0}(0)} \varepsilon_{\tau}(-\mathrm{i})^{\tau} J_{\tau}^{\prime}\left(k_{0} R\right) \cos (\tau \beta), & \chi=\mathrm{S}, \\
0, & \chi=\mathrm{R}\end{cases} \\
X_{\tau, \zeta}^{(2)}=\left\{\begin{array}{ll}
\frac{\tau}{R}, \quad \zeta=0 \\
\frac{\beta_{\zeta} I_{\tau}^{\prime}\left(\beta_{\zeta} R\right)}{I_{\tau}\left(\beta_{\zeta} R\right)}, & \zeta \neq 0
\end{array} ; \quad Y_{\tau, \zeta}^{(2)}= \begin{cases}\frac{1}{R}, & \zeta=0, \tau=0 \\
-\frac{\tau}{R}, & \zeta=0, \tau \neq 0, \\
\frac{\beta_{\zeta} K_{\tau}^{\prime}\left(\beta_{\zeta} R\right)}{K_{\tau}\left(\beta_{\zeta} R\right)}, & \zeta \neq 0\end{cases} \right.
\end{gathered}
$$




$$
Z_{\tau, \zeta}^{(2)}= \begin{cases}\frac{k_{0} H_{\tau}^{\prime}\left(k_{0} R\right)}{H_{\tau}\left(k_{0} R\right)}, & \zeta=0 \\ \frac{k_{\zeta} K_{\tau}^{\prime}\left(k_{\zeta} R\right)}{K_{\tau}\left(k_{\zeta} R\right)}, & \zeta=1,2,3 \cdots\end{cases}
$$

After inserting Eqs. (16) and (22) into Eq.(31), multiplying both sides by $\cos \left[\beta_{\zeta}(z+h)\right] \cos (\tau \theta)$ and integrating for $z \in[-h,-d]$ and $\theta \in[0, \pi]$, for any pair of integer $(\tau, \zeta)$, it can be obtained that

$$
\begin{aligned}
& \sum_{l=0}^{\infty}\left(\frac{\pi}{2}\left(\frac{\tilde{I}_{\tau}\left(k_{l} R_{\mathrm{i}}\right)}{k_{l} \tilde{I}_{\tau}^{\prime}\left(k_{l} R_{\mathrm{i}}\right)} A_{\tau, l}^{\chi}+\frac{\tilde{I}_{-\tau}\left(k_{l} R_{\mathrm{i}}\right)}{k_{l} \tilde{I}_{-\tau}^{\prime}\left(k_{l} R_{\mathrm{i}}\right)} A_{-\tau, l}^{\chi}\right)-\mathrm{i} \sum_{\substack{m=-\infty \\
m \neq \pm \tau}}^{\infty} \frac{m\left[(-1)^{m-\tau}-1\right]}{m^{2}-\tau^{2}} \frac{\tilde{I}_{m}\left(k_{l} R_{\mathrm{i}}\right)}{k_{l} \tilde{I}_{m}^{\prime}\left(k_{l} R_{\mathrm{i}}\right)} A_{m, l}^{\chi}\right) L_{\zeta, l}, \\
& =\frac{\pi(h-d)}{\varepsilon_{\tau} \varepsilon_{\zeta}}\left(X_{\tau, \zeta}^{(3)} C_{\tau, \zeta}^{\chi}+Y_{\tau, \zeta}^{(3)} D_{\tau, \zeta}^{\chi}\right)+f_{3}^{\chi}
\end{aligned}
$$

where

$$
\begin{aligned}
& f_{3}^{\chi}=\left\{\begin{array}{ll}
0, & \chi=\mathrm{S} \\
\frac{\delta_{\tau, 0} \delta_{\zeta, 0} \mathrm{i} \pi(h-d)}{\rho \omega}, & \chi=\mathrm{R}
\end{array},\right. \\
& X_{m, l}^{(3)}=\left\{\begin{array}{l}
\left(\frac{R_{\mathrm{i}}}{R}\right)^{m}, \quad l=0 \\
\frac{I_{m}\left(\beta_{l} R_{\mathrm{i}}\right)}{I_{m}\left(\beta_{l} R\right)}, \quad l \neq 0
\end{array} ; \quad Y_{m, l}^{(3)}=\left\{\begin{array}{l}
1+\ln \left(\frac{R_{\mathrm{i}}}{R}\right), \quad l=0, m=0 \\
\left(\frac{R}{R_{\mathrm{i}}}\right)^{m}, \quad l=0, m \neq 0 \\
\frac{K_{m}\left(\beta_{l} R_{\mathrm{i}}\right)}{K_{m}\left(\beta_{l} R\right)}, \quad l \neq 0
\end{array} .\right.\right.
\end{aligned}
$$

After inserting Eqs. (22) and (25) into Eq. (32), multiplying both sides by $\cos \left[\beta_{\zeta}(z+h)\right] \cos (\tau \theta)$ and integrating for $z \in[-h,-d]$ and $\theta \in[0, \pi]$, for any pair of integer $(\tau, \zeta)$, we have

$$
\frac{h-d}{\varepsilon_{\zeta}}\left(C_{\tau, \zeta}^{\chi}+D_{\tau, \zeta}^{\chi}\right)-\sum_{l=0}^{\infty} E_{\tau, l}^{\chi} L_{\zeta, l}=f_{4}^{\chi},
$$

where

$$
f_{4}^{\chi}=\left\{\begin{array}{ll}
-\frac{2 \varepsilon_{\tau} \mathrm{i} g A L_{\zeta, 0}}{\omega Z_{0}(0)}(-\mathrm{i})^{\tau} J_{\tau}\left(k_{0} R\right) \cos (\tau \beta), & \chi=\mathrm{S} \\
0, & \chi=\mathrm{R}
\end{array} .\right.
$$

Eqs.(A.1), (A.4), (A.8) and (A.11) form a linear algebraic system, which can be used to solve $A_{m, l}^{\chi}, C_{m, l}^{\chi}, D_{m, l}^{\chi}$ and $E_{m, l}^{\chi}$ numerically after truncation. In the present model, the infinite terms of $\mathrm{e}^{\mathrm{i} m \theta} / \cos (m \theta)$, and $Z_{l}(z) / \cos \left[\beta_{l}(z+h)\right]$ are truncated at $m=M$ and $l=L$, respectively. Accurate results can be obtained by choosing $M=12, L=20$. 


\section{References}

[1] Fleming AN, Macfarlane GJ. Experimental flow field comparison for a series of scale model oscillating water column wave energy converters. Mar Struct. 2017;52:108-25.

[2] Sheng W, Alcorn R, Lewis A. Assessment of primary energy conversions of oscillating water columns.

I. Hydrodynamic analysis. J Renew Sustain Ener. 2014;6:053113.

[3] Guo B, Patton R, Jin S, Gilbert J, Parsons D. Nonlinear modeling and verification of a heaving point absorber for wave energy conversion. leee T Sustain Energ. 2018;9:453-61.

[4] Zheng S, Zhang Y. Analysis for wave power capture capacity of two interconnected floats in regular waves. J Fluid Struct. 2017;75:158-73.

[5] Renzi E, Dias F. Hydrodynamics of the oscillating wave surge converter in the open ocean. European Journal of Mechanics B/Fluids. 2013;41:1-10.

[6] Fernandez H, Iglesias G, Carballo R, Castro A, Fraguela JA, Taveira-Pinto F, et al. The new wave energy converter WaveCat: Concept and laboratory tests. Mar Struct. 2012;29:58-70.

[7] Drew B, Plummer AR, Sahinkaya MN. A review of wave energy converter technology. P I Mech Eng a-J Pow. 2009;223:887-902.

[8] Falnes J. A review of wave-energy extraction. Mar Struct. 2007;20:185-201.

[9] Mustapa MA, Yaakob OB, Ahmed YM, Rheem CK, Koh KK, Adnan FA. Wave energy device and breakwater integration: A review. Renew Sust Energ Rev. 2017;77:43-58.

[10] Astariz S, Iglesias G. The economics of wave energy: A review. Renew Sust Energ Rev. 2015;45:397-408.

[11] Heras-Saizarbitoria I, Zamanillo I, Laskurain I. Social acceptance of ocean wave energy: A case study of an OWC shoreline plant. Renew Sust Energ Rev. 2013;27:515-24.

[12] Ning DZ, Zhao XL, Chen LF, Zhao M. Hydrodynamic performance of an array of wave energy converters integrated with a pontoon-type breakwater. Energies. 2018;11:685.

[13] Perez-Collazo C, Greaves D, Iglesias G. Hydrodynamic response of the WEC sub-system of a novel hybrid wind-wave energy converter. Energy Conversion and Management. 2018;171:307-25.

[14] Malara G, Gomes RPF, Arena F, Henriques JCC, Gato LMC, Falcão AFO. The influence of three-dimensional effects on the performance of U-type oscillating water column wave energy harvesters. Renew Energ. 2017;111:506-22.

[15] Evans DV, Porter R. Hydrodynamic characteristics of an oscillating water column device. Appl Ocean Res. 1995;17:155-64.

[16] Morris-Thomas MT, Irvin RJ, Thiagarajan KP. An investigation into the hydrodynamic efficiency of an oscillating water column. Journal of Offshore Mechanics and Arctic Engineering. 2007;129:273-8.

[17] Elhanafi A, Fleming A, Macfarlane G, Leong Z. Underwater geometrical impact on the hydrodynamic performance of an offshore oscillating water column-wave energy converter. Renew Energ. 2017;105:209-31.

[18] Sheng W, Alcorn R, Lewis A. Assessment of primary energy conversions of oscillating water columns. II. Power take-off and validations. J Renew Sustain Ener. 2014;6:053114.

[19] López I, Iglesias G. Efficiency of OWC wave energy converters: A virtual laboratory. Appl Ocean Res. 2014;44:63-70.

[20] López I, Pereiras B, Castro F, Iglesias G. Performance of OWC wave energy converters: influence of turbine damping and tidal variability. Int J Energ Res. 2015;39:472-83.

[21] Pereiras B, López I, Castro F, Iglesias G. Non-dimensional analysis for matching an impulse turbine to an OWC (oscillating water column) with an optimum energy transfer. Energy. 2015;87:481-9. 
[22] Elhanafi A, Fleming A, Macfarlane G, Leong Z. Numerical energy balance analysis for an onshore oscillating water column-wave energy converter. Energy. 2016;116:539-57.

[23] Malara G, Arena F. Analytical modelling of an U-Oscillating Water Column and performance in random waves. Renew Energ. 2013;60:116-26.

[24] Malara G, Romolo A, Fiamma V, Arena F. On the modelling of water column oscillations in U-OWC energy harvesters. Renew Energ. 2017;101:964-72.

[25] Ning D, Wang R, Zhang C. Numerical simulation of a dual-chamber oscillating water column wave energy converter. Sustainability. 2017;9.

[26] He F, Huang Z, Law AWK. An experimental study of a floating breakwater with asymmetric pneumatic chambers for wave energy extraction. Applied Energy. 2013;106:222-31.

[27] He F, Leng J, Zhao XZ. An experimental investigation into the wave power extraction of a floating box-type breakwater with dual pneumatic chambers. Appl Ocean Res. 2017;67:21-30.

[28] Howe D, Nader JR. OWC WEC integrated within a breakwater versus isolated: Experimental and numerical theoretical study. International Journal of Marine Energy. 2017;20:165-82.

[29] Falcão AFO, Henriques JCC, Gato LMC. Air turbine optimization for a bottom-standing oscillating-water-column wave energy converter. Journal of Ocean Engineering and Marine Energy. 2016;2:459-72.

[30] Nader JR. Interaction of ocean waves with oscillating water column wave energy convertors. Wollongong: University of Wollongong; 2013.

[31] Martins-rivas $\mathrm{H}$, Mei CC. Wave power extraction from an oscillating water column at the tip of a breakwater. J Fluid Mech. 2009;626:395-414.

[32] Martins-rivas $\mathrm{H}$, Mei CC. Wave power extraction from an oscillating water column along a straight coast. Ocean Eng. 2009;36:426-33.

[33] Lovas S, Mei CC, Liu YM. Oscillating water column at a coastal corner for wave power extraction. Appl Ocean Res. 2010;32:267-83.

[34] Sarmento AJNA, Falcão AFdO. Wave Generation by an Oscillating Surface-Pressure and Its Application in Wave-Energy Extraction. J Fluid Mech. 1985;150:467-85.

[35] Zheng S, Zhang $Y$, Iglesias G. Wave-structure interaction in hybrid wave farms. J Fluid Struct. 2018;83:386-412.

[36] Zheng S, Zhang Y. Theoretical modelling of a new hybrid wave energy converter in regular waves. Renew Energ. 2018;128A:125-41.

[37] Zheng S, Zhang Y. Wave diffraction from a truncated cylinder in front of a vertical wall. Ocean Eng. 2015;104:329-43.

[38] Zheng S, Zhang Y. Wave radiation from a truncated cylinder in front of a vertical wall. Ocean Eng. 2016;111:602-14.

[39] Falnes J. Ocean waves and oscillating systems: Linear interaction including wave-energy extraction. Cambridge, UK: Cambridge University Press; 2002. 